\title{
Feasibility, Safety and Diagnostic Yield of Omom CE, a New Capsule Endoscopy System: The First Experience in Caucasian Patients
}

\author{
Flaminia Cavallaro ${ }^{1 *}$, Gian Eugenio Tontini ${ }^{1}$, Rosamaria Bozzi ${ }^{2}$, Nicoletta Nandi ${ }^{1}$, Domenico \\ Cattaneo $^{2}$, Federica Villa ${ }^{3}$, Sergio Cadoni ${ }^{4}$, Paolo Gallittu ${ }^{4}$, Emanuele Rondonotti ${ }^{5}$ and Maurizio \\ Vecchi $^{1,6}$ \\ ${ }^{1}$ Gastroenterology \& Digestive Endoscopy Unit, IRCCS Policlinico San Donato, Milano, Italy \\ ${ }^{2}$ Surgery \& Digestive Endoscopy Unit, V. Monaldi Hospital, Naples, Italy \\ ${ }^{3}$ Gastroenterology \& Digestive Endoscopy Unit, C.Cantù Hospital, AO Legnano, Abbiategrasso, Italy \\ ${ }^{4}$ Digestive Endoscopy Unit, Santa Barbara Hospital, Iglesias, Italy \\ ${ }^{5}$ Gastroenterology Unit, Valduce Hospital, Como, Italy \\ ${ }^{6}$ Department of Biomedical Sciences for Health, University of Milan, Milano, Italy
}

Received: May 11, 2017; Accepted: July 19, 2017; Published: August 21, 2017

${ }^{*}$ Corresponding author: Flaminia Cavallaro, Gastroenterology and Digestive Endoscopy Unit, IRCCS Policlinico San Donato, Milano, Italy, E-mail: flaminia.cavallaro@gmail.com

\begin{abstract}
Capsule endoscopy, since its FDA approval in 2001, has dramatically changed the study of the small bowel. However, one of the main limitations to its diffusion is the relatively high cost it has. More recently, a new video capsule system (OMOM CE) has been developed in China and obtained the CE mark. Aim of the study was to assess the feasibility, safety and diagnostic yield of OMOM CE. 118 consecutive patients with suspected small bowel disease underwent OMOM CE. Indications to the exam were obscure gastrointestinal bleeding, known or suspected Crohn's disease, suspected small bowel tumor, familial adenomatous polyposis. All patients ingested the capsule easily. Visualization of the entire small bowel was achieved in 114patients (97\%) and capsule retention without obstruction occurred in 1 patient due to a previously undiagnosed Crohn's disease stricture (0.8\%). The overall diagnostic yield was $48 \%$. In our series, the overall diagnostic yield was similar to that reported in the literature. OMOM CE appears to be a practical, safe, easy to perform procedure, providing a similar diagnostic yield and an even superior observation of the small bowel compare to other systems. Its lower cost might also encourage its diffusion.
\end{abstract}

\section{Introduction}

Small bowel evaluation has been considered for a long time technically challenging because of its length, location and tortuosity. But in 2001, with the FDA approval of capsule endoscopy started a revolution in the study of small bowel that, in the last years, has progressively and rapidly expanded [1-5]. Nevertheless, one of the main limitations to the spread of capsule endoscopy is the high cost and therefore a questionable cost-effectiveness when used in clinical practice. Lately the picture could change to the introduction in the European of a new video capsule system (OMOM CE) produced by Jinshan Science \& Technology Company (Chongqing, China) [6,7]; this device has and obtained the CE mark for marketing in Europe. Indeed, this device costs approximately half the other capsule systems. However, to date only few studies have been performed on the use in clinical practice of this new video capsule system, and none of them was carried out in the western world.
This study aimed to assess the feasibility, safety and diagnostic yield of the OMOM CE in several clinical settings investigating possible small bowel disease conditions.

\section{Patients and Methods}

\section{Patients}

The total recruited patients were 118 ( 61 men, 57 women, mean age 53 years, range 18-86 years), all with suspected small bowel pathology. Patients underwent OMOM CE in one of the three Gastroenterology Unit involved in the study (Gastroenterology \& Digestive Endoscopy Unit, IRCCS Policlinico San Donato - University of Milan; Surgery \& Digestive Endoscopy Unit, V. Monaldi Hospital, Naples; Santa Barbara Hospital, Iglesias).

Indications to perform the exam were: obscure gastrointestinal bleeding, known or suspected Crohn's disease, suspected small bowel tumor and familial adenomatous polyposis. The distribution of patients for each diagnostic subgroup is reported in table 1 . All the included subjects had previously performed other investigations, such as esophagogastroduodenoscopy, colonoscopy, small bowel follow through, enteroclysis, abdominal computed tomography or magnetic resonance.

\begin{tabular}{|c|c|}
\hline Table 1: Indications for capsule endoscopy & \\
\hline Indications Of Patients For Capsule Endoscopy & Number \\
\hline Obscure gastrointestinal bleeding & 55 \\
\hline Known or suspected Crohn's disease & 57 \\
\hline Familial adenomatous polyposis & 3 \\
\hline Suspected small bowel tumor & 3 \\
\hline Total patients & 118 \\
\hline
\end{tabular}

\section{Methods}

The device used in all the enrolled patients was the OMOM capsule endoscopy (Jinshan Science \& Technology Company, Chongqing, China). The system is composed of three parts: a disposable capsule, an image recorder jacket and an image workstation. 
In the recorder jacket, there are 14 receiver elements placed close to the abdomen and waist. The images acquired are transmitted from the capsule to the recorder via a digital radio frequency communication channel. Moreover, it is included a portable real-time monitor device that allows the endoscopist not only to follow the progression of the capsule, but also to send possible commands to the OMOM system to modify the rate of frame $(2,1$ or $0.5 \mathrm{fps})$, the flash intensity and the capsule state (sleep or awake). The recorder is later connected to the workstation, in order to download and process the images.

\begin{tabular}{|c|c|c|c|c|}
\hline \multicolumn{5}{|l|}{ Table 2: Diagnostic yield } \\
\hline Indication & Nr & Positive & Suspicious & Negative \\
\hline $\begin{array}{c}\text { Obscure GI bleeding } \\
\begin{array}{c}\text { Suspected or known } \\
\text { Crohn's disease }\end{array}\end{array}$ & 55 & $33(60 \%)$ & $9(16 \%)$ & $13(24 \%)$ \\
\hline FAP & 3 & $1(33 \%)$ & $0(0 \%)$ & $2(67 \%)$ \\
\hline $\begin{array}{c}\text { Suspected small bowel } \\
\text { tumor }\end{array}$ & 3 & $2(67 \%)$ & $0(0 \%)$ & $1(33 \%)$ \\
\hline Total & 118 & $56(48 \%)$ & $12(10 \%)$ & $50(42 \%)$ \\
\hline
\end{tabular}

OMOM capsule endoscopy differs from the other available capsule endoscopy systems in dimensions, as the OMOM CE is slightly bigger, the presence of an antenna-carrying jacket, the possibility to modify the image acquisition rate and, last but not least, its significantly lower cost. The main features of the system are shown in figures 1-3. The day before the procedure, all the subjects followed a clear semi-liquid diet and assumed 2 L of PEG (polyethylene glycol solution) in the afternoon.

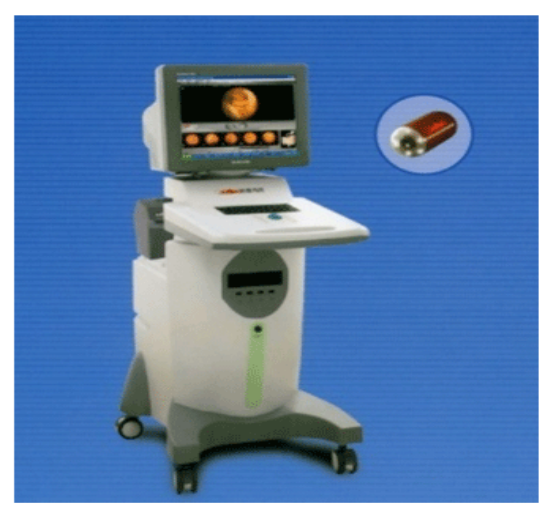

Figure 1: Workstation of the system

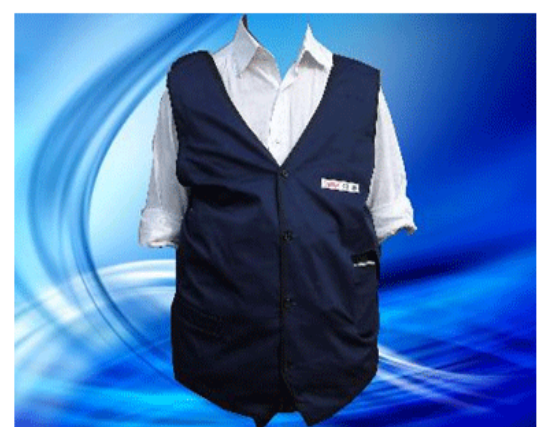

Figure 2: Antenna carrying jacket

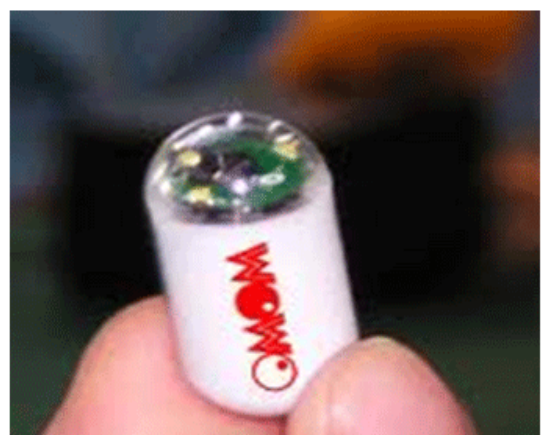

Figure 3: Video capsule in front-lateral view
Additionally, in one of the three centres, in the first hour of examination, the real-time monitor was used to assess capsule location. If the capsule, after 60 minutes from ingestion, did not pass the pylorus reaching the small bowel, then metoclopramide $10 \mathrm{mg}$ was administered to the patient intravenously. The acquired images were reviewed by two expert gastroenterologists that according to the findings classified the videos as diagnostic, suspicious or negative.

\section{Statistical analysis}

Quantitative variables were expressed as mean \pm SD values. Fisher's test was used to compare occult OGIB and overt OGIB diagnostic yield.

\section{Results}

All patients ingested the OMOM CE very easily and no complications were observed. All data analyzed were normally distributed. The recording time was 420 to 580 minutes (mean time $514 \mathrm{~min}$, SD 39). Surprisingly, the mean pyloric transit time -defined as the recorded time at the first image of duodenum- was 78 minutes (SD 44) in patients who received metoclopramide while in those not receiving it, was 27 minutes (SD 16). For what concerns the mean small bowel transit time - defined as the time from the first duodenal image to the time of the first cecal image- was 241 minutes (SD 123) in patients who received metoclopramide and 235 minutes (SD 73) in those who did not receive it. Cases in which the capsule was not able to reach the cecumwere excluded from analysis of small bowel transit time.

Complete visualization of the small bowel was achieved in 114 patients (97\%). Capsule retention without obstruction occurred in only 1 patient $(0.8 \%)$ due to a previously undiagnosed Crohn's disease stricture at the terminal ileum, in a patient with diarrhoea and without any obstructive symptom. This patient underwent surgical treatment of the stricture and capsule recovery. In 4 patients the capsule did not reach the cecum within the time of recording. In 3 of them the capsule failed to reach the cecum because of the impact with a lesion (a jejunal stricture due to a previously unknown Crohn's disease in one patient, an ileal mass in another one and a duodenal substenosis in the last case); in the fourth patient, an actual cause could not be found, as the only pathological finding was angiodysplasia. Anyway, they all spontaneously expelled the capsule in 10 days, except for the patient with the jejunal stricture, whom experienced symptomless retention and had to undergo surgical treatment, allowing also for capsule retrieval.

The overall diagnostic yield, when considering only positive findings, was $48 \%$. If suspicious findings were included as well, the diagnostic yield increased up to $58 \%$. The diagnostic yield observed in the different subgroups is shown in table 2. Diagnostic yield in patients with OGIB was $76 \%$ (when counting both positive and suspicious findings), which is greater if compared to that of the nonOGIB subgroup. This finding further confirms that OGIB is the most important indication for capsule endoscopy. When patients were divided according to the type of bleeding (overt vs. occult), the diagnostic yield of OGIB was similar ( $p=0$, 7) to that of non-OGIB as reported in table 3.

Table 3: Diagnostic yield in OGIB

\begin{tabular}{|l|l|l|l|l|}
\hline Indication & $\mathbf{N r}$ & Positive & Suspicious & Negative \\
\hline OGIB Occult & 34 & $20(59 \%)$ & $6(18 \%)$ & $8(23 \%)$ \\
\hline OGIB Overt & 21 & $14(67 \%)$ & $1(5 \%)$ & $6(28 \%)$ \\
\hline
\end{tabular}

Citation: Cavallaro F, Tontini GE, Bozzi R, et al. (2017) Feasibility, Safety and Diagnostic Yield of Omom CE, a New Capsule Endoscopy System: The First Experience in Caucasian Patients. Gastroenterol Pancreatol Liver Disord 5(1):1-4. DOI: http://dx.doi.org/10.15226/2374-815X/5/1/001103 


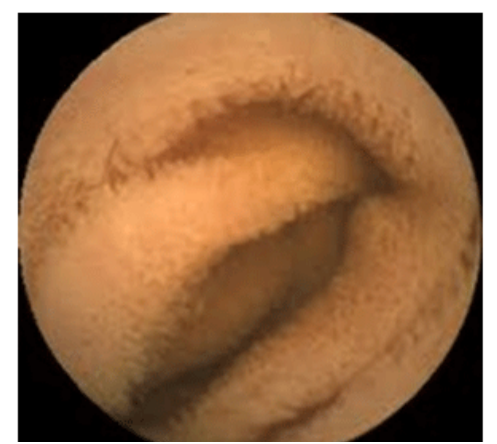

Figure 4: Normal jejunum

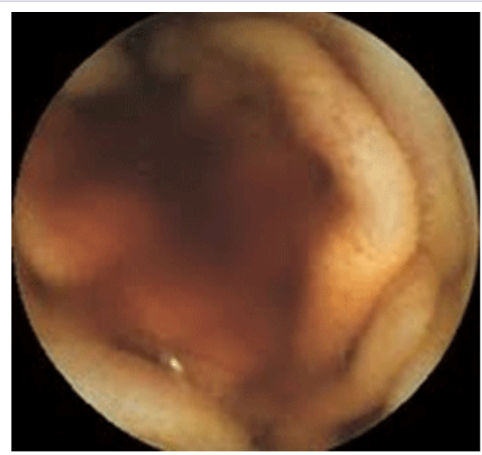

Figure 5: Red blood in the lumen

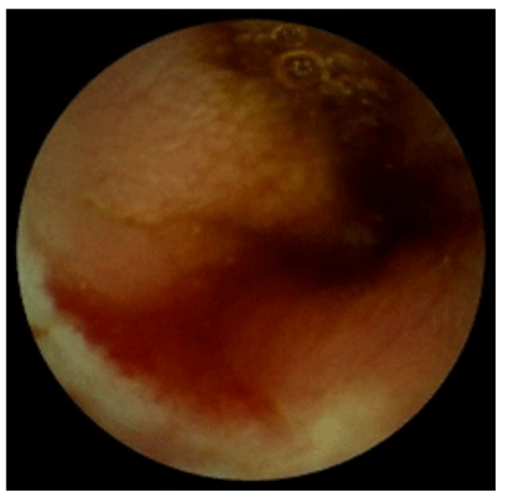

Figure 6: Jejunal ulcer

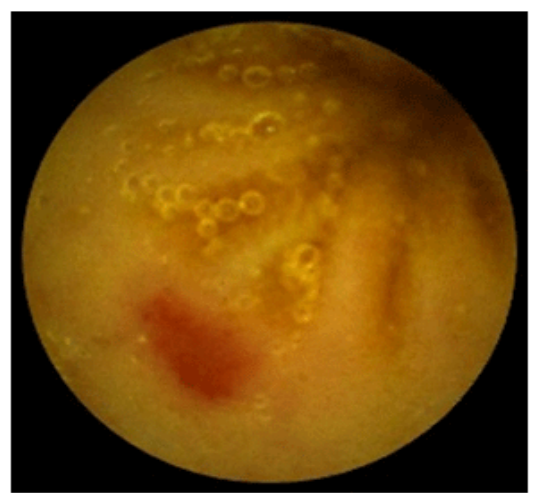

Figure 7: Ilealangiodysplasia

Table 4: Small-bowel finding in positive patients

\begin{tabular}{|c|c|c|c|}
\hline Findings & Overall & OGIB & Non OGIB \\
\hline Angioectasia & 15 & 15 & 0 \\
\hline Ulcer & 20 & 14 & 6 \\
\hline Erosion & 6 & 2 & 5 \\
\hline Polyp & 4 & 3 & 1 \\
\hline Active bleeding & 6 & 6 & 0 \\
\hline Stricture & 3 & 1 & 1 \\
\hline Villousatrophy & 2 & 0 & 1 \\
\hline Tumor & 2 & 0 & 1 \\
\hline
\end{tabular}

one patient had two types of lesions
Angiodysplasias were the most common findings [8], being observed in all subjects in the OGIB subgroup. Other possible associated findings included ulcers, erosions, polyps, active bleeding with no recognizable lesion and smallAngiodysplasias were the most common findings [8], being observed in all subjects in the OGIB subgroup. Other possible associated findings included ulcers, erosions, polyps, active bleeding with no recognizable lesion and small-bowel tumors. All the findings are reported in table 4. The main findings are shown in figures 4-7.

\section{Discussion}

Since the development of the first model of capsule endoscopy, there has been a continuous technological progress and consequent technical advancement that has allowed capsule endoscopy to become a powerful tool for the evaluation of suspected or known small bowel disease conditions. However, in times of strict cost containment, the high cost of this procedure has represented one the main limitations on its use. Recently, a relatively low-cost capsule endoscopy was developed and has been used in large patients' population studies in China. This is the first study evaluating the overall performance of OMOM capsule endoscopy in a group of patients of Caucasian origin.

In our hands, the system proved to be easy to use and safe. Indeed, retention without obstruction occurred in only 1 patient and it was due to a previously undiagnosed Crohn's disease stricture at the level of the terminal ileum. Retention was symptomless and the patient underwent surgical treatment of the stricture and capsule recovery. Some of the features of the OMOM system have revealed to be extremely useful in making the procedure more adjustable and tailored to the many potential clinical settings. In particular, the possibility of modulating the flash intensity and the ON/OFF status of the capsule are unique of this system. While the first one might be convenient in conditions of low visibility (in the stomach or if there are residues or blood in the lumen); the second one may be helpful in saving battery life when a distal lesion has to be reached. Another important feature is the possibility of monitoring the pyloric transit in order to decide whether or not to use a prokinetic to fasten up the process and achieve exam completion; indeed, it is known that delayed gastric time is one of the most frequent causes of failure to reach the cecum $[9,10]$.

In our series, OMOM capsule endoscopy reached the cecum in most of patients (97\%). Our result is much higher than the figures so far reported in literature for all the other capsule systems [11-13]. Even though the use of prokinetics may explain this result $[10,14]$ this does not seem to be the case in our study. In fact, when are considered patients from the centers neither utilizing prokinetics injection nor real-time viewer, the proportion of capsule reaching the cecum was even higher (37/38 patients, 97\%). Therefore, the role of these factors can be easily ruled out. The OMOM CE is slightly bigger and heavier than the other capsules and this may favor a relatively faster progression along the small bowel. Moreover, our patient series was characterized by a relatively large proportion of patients with clinical conditions, such as diarrhea and overt OGIB, possibly associated with accelerated peristalsis and a short small bowel transit time. As a matter of fact, the small bowel transit time observed in the present study is quite short but substantially similar to that reported in the literature.

This might be explained considering that transit speed can be affected by multiple variables, such as the completion rate, the definition of the small bowel transit time used (many Authors include also patients in whom the capsule never reached the cecum), the age of patients, the comorbidities and drugs assumption that may affect bowel peristalsis (like diabetes or neuropathies, and opiods or prokinetics, respectively), the in-patient or out-patient settings, etc. Another possible explanation for this result could be the relatively longer lifespan of OMOM capsule endoscopy battery that allows for prolonged recording. In any cases, a more complete visualization of the small bowel could be crucial to obtain an even higher diagnostic yield than that achieved by currently available devices.

In our series, the diagnostic yield was assessed considering only positive 
findings (see table 2); the results were similar to those reported in literature, with an overall detection rate of $48 \%$. In particular, it was $60 \%$ in OGIB and $35 \%$ in known or suspected Crohn's disease. In agreement with previously described studies, the diagnostic yield of capsule endoscopy varies depending on the indication for which it is performed, being about $50 \%$ for OGIB in a recent series [11,15-16] while, for suspected Crohn's disease, widely ranges between 33-70\% [16-18]. The results of the present study are quite encouraging, as they show diagnostic figures at least equal to those reported in the literature, despite the fact that a statistical comparison is not feasible due to the relatively small number of patients evaluated in our study.

\section{Conclusion}

In our series, OMOM capsule endoscopy appears to be a practical, safe and easy to perform procedure in the evaluation of small bowel disorders. Compared to the other capsule systems on the market, OMOM capsule provides similar overall diagnostic yields and longer time of observation of the small bowel.

Furthermore, its significantly lower cost, with respect to all other systems available in Europe, should encourage its diffusion because of the superior cost/ effectiveness ratio.

\section{References}

1. Iddan G, Meron G, Glukhovsky A, Swain P. Wireless capsule endoscopy. Nature. 2000;405-417.

2. Mishkin DS, Chuttani R, Croffie J, Disario J, Liu J, Shah R, et al. ASGE Technology Status Evaluation Report: wireless capsule endoscopy. Gastrointest Endosc. 2006;63(4):539 545. DOI: 10.1016/j.gie.2006.01.014

3. Pennazio M, Eisen G, Goldfarb N. ICCE consensus for obscure gastrointestinal bleeding Endoscopy. 2005;37(10):1046-1050. DOI: 10.1055/s-2005-870319

4. Mergener K, Ponchon T, Gralnek I, Pennazio M, Gay G, Selby W, et al. Literature review and recommendations for clinical application of small-bowel capsule endoscopy, based on a panel discussion by international experts. Consensus statements for small-bowe capsule endoscopy, 2006/2007. Endoscopy. 2007;39(10):895-909. DOI: 10.1055/s2007-966930

5. Ladas SD, Triantafyllou K, Spada C, Riccioni ME, Rey JF, Niv Y, et al. ESGE Clinical Guidelines Committee. European Society of Gastrointestinal Endoscopy (ESGE) recommendations (2009) on clinical use of video capsule endoscopy to investigate small-bowel, esophageal and colonic diseases. Endoscopy. 2010;42(3):220-227. DOI: $10.1055 / \mathrm{s}-0029-1243968$

6. Liao Z, Gao R, Li F, Xu C, Zhou Y, Wang JS, et al. Fields of applications, diagnostic yields and findings of OMOM capsule endoscopy in 2400 Chinese patients. World J gastroenterol. 2010;16(21):2669-2676
7. Li C, Zhang B, Chen C, Li YM. OMOM capsule endoscopy in diagnosis of small bowel disease. J Zhejiang Univ Sci B. 2008;9(11):857-862. Doi: 10.1631/jzus.B0820034

8. Pennazio M, Santucci R, RondonottiE, Abbiati C, Beccari G, Rossini FP, et al. Outcome of patients with obscure gastrointestinal bleeding after capsule endoscopy: report of 100 consecutive cases. Gastroenterology. 2004;126(3):643-653.

9. Ogata H, Kumai K, Imaeda H, Aiura K, Hisamatsu T, Okamoto S, et al. Clinical impact of a newly developed capsule endoscope: usefulness of a real-time image viewer for gastric transit abnormality. J Gastroenterol. 2008;43(3):186-192. Doi: 10.1007/s00535-0072140-y

10.Postgate A, Tekkis P, Patterson N, Fitzpatrick A, Bassett P, Fraser C. Are bowel purgatives and prokinetics useful for small-bowel capsule endoscopy? A prospective randomized controlled study. Gastrointest Endosc. 2009;69(6):1120-1128. doi: 10.1016/j. gie.2008.06.044

11.Liao Z, Gao R, Xu C, Li ZS. Indications and detection, completion, and retention rates of small-bowel capsule endoscopy: a systematic review. Gastrointest Endosc. 2010;71(2):280-286. Doi: 10.1016/j.gie.2009.09.031

12.Selby W. Complete small-bowel transit in patients undergoing capsule endoscopy: determinating factors and improvement with metoclopramide. Gastrointest Endosc. 2005;61(1):80-85.

13.Rondonotti E, Herrerias J, Pennazio M, Caunedo A, Mascarenhas-Saraiva M, de Franchis R. Complications, limitations, and failures of capsule endoscopy: a review of 733 cases. Gastrointestinal Endoscopy. 2005;62(5):712-716.

14.Westerhof J, Weersma RK, Koornstra JJ. Risk factors for incomplete small-bowel capsule endoscopy. GastrointestinalEndoscopy. 2009;69(1):74-80. Doi: 10.1016/j. gie.2008.04.034

15.Estévez E, Gonzalez-Conde B, Vazquez-Iglesias JL, de Los Angeles Vázquez-Millán M, Pértega S, Alonso PA, et al. Diagnostic detection rate and clinical outcomes after capsule endoscopy in 100 consecutive patients with obscure gastrointestinal bleeding. Eur J Gastroenterol Hepatol. 2006;18(8):881-888.

16. Rondonotti E, Villa F, Mulder CJ, Jacobs MA, de Franchis R. Small bowel capsule endoscopy in 2007: indications, risk and limitations. World J Gastroenterol. 2007;13(46):61406149.

17.Marmo R, Rotondano G, Piscopo R, Bianco MA, Siani A, Catalano O, et al. Capsule endoscopy versus enteroclysis in the detection of small bowel involvement in Crohn's disease: a prospective trial. Clin Gastroenterol Hepatol. 2005;3(8):772-776.

18.Triester SL, Leighton JA, Leontiadis GI, Gurudu SR, Fleischer DE, Hara AK, et al. A metaanalysis of the yield of capsule endoscopy compared to other diagnostic modalities in patients with non-stricturing small bowel Crohn's disease. Am J Gastroenterol. 2006;101(5):954-964. 